\title{
CR-WARPED PRODUCT SUBMANIFOLDS OF NEARLY KAEHLER MANIFOLDS
}

\author{
Nadia S. Al-Luhaibi, Falleh R. Al-Solamy, and Viqar Azam Khan
}

\begin{abstract}
As warped product manifolds provide an excellent setting to model space time near black holes or bodies with large gravitational field, the study of these manifolds assumes significance in general. B. Y. Chen [4] initiated the study of CR-warped product submanifolds in a Kaehler manifold. He obtained a characterization for a CR-submanifold to be locally a CR-warped product and an estimate for the squared norm of the second fundamental form of CR-warped products in a complex space form (cf [6]). In the present paper, we have obtained a necessary and sufficient conditions in terms of the canonical structures $P$ and $F$ on a CR-submanifold of a nearly Kaehler manifold under which the submanifold reduces to a locally CR-warped product submanifold. Moreover, an estimate for the second fundamental form of the submanifold in a generalized complex space is obtained and thus extend the results of Chen to a more general setting.
\end{abstract}

\section{Introduction}

R. L. Bishop and B. O'Neill [1] introduced the notion of warped product manifolds by homothetically warping the product metric of a product manifold $B \times F$ onto the fibers $p \times F$ for each $p \in B$. This generalized product metric appears in differential geometric studies in a natural way. For instance a surface of revolution is a warped product manifold. Moreover, many important submanifolds in real and complex space forms are expressed as warped product submanifolds. In view of its physical applications many research articles have recently appeared exploring existence (or non existence) of warped product submanifolds in known spaces (cf [8], [13], etc). B. Y. Chen [4] initiated the investigations by showing that there does not exist a warped product CR-submanifold $N_{\perp} \times_{f} N_{T}$ in a Kaehler manifold. B. Sahin [11], extending the result of Chen proved that there exists no semi-slant warped product submanifolds in a Kaehler manifold other than CR-warped product submanifolds $N_{T} \times_{f} N_{\perp}$, where $N_{T}$ and $N_{\perp}$ are holomorphic and totally real submanifolds

Received November 24, 2007.

2000 Mathematics Subject Classification. 53C40, 53C15, 53C42.

Key words and phrases. nearly Kaehler manifold, CR-submanifold, CR-warped product submanifold, canonical structure, generalized complex space form. 
of the underlying Kaehler manifold. Interesting geometric properties of CRwarped product submanifolds are obtained as well as many examples of these warped product submanifolds are provided in [4]. In view of the interesting geometric features of a nearly Kaehler manifolds and the non existence of CRproducts in $S^{6}$ (cf [14]), it is worthwhile to study CR-warped products in a nearly Kaehler manifold. In the present paper, we have worked out conditions under which a CR-submanifold reduces to locally a CR-warped product submanifold. Moreover, an inequality for the squared norm of the second fundamental form of CR-warped product submanifolds in a generalized complex space form is obtained.

\section{Preliminaries}

Let $\bar{M}$ be an almost Hermitian manifold with an almost complex structure $J$ and a Hermitian metric $g$, i.e.,

$$
J^{2}=-I, \quad \text { and } \quad g(J U, J V)=g(U, V)
$$

for all vector fields $U, V$ on $\bar{M}$. If $J$ is parallel with respect to the Levi-Civita connection $\bar{\nabla}$ on $\bar{M}$, i.e., $\bar{\nabla} J=0$, then $(\bar{M}, J, g, \bar{\nabla})$ is called a Kaehler manifold. A more general structure on $\bar{M}$, namely nearly Kaehler structure is defined by a weaker condition namely

$$
\left(\bar{\nabla}_{U} J\right) V+\left(\bar{\nabla}_{V} J\right) U=0 .
$$

A necessary and sufficient condition for a nearly Kaehler manifold to be a Kaehler manifold is the vanishing of the Nijenhuis tensor of $J$. Any four dimensional nearly Kaehler manifold is a Kaehler manifold. A typical example of a nearly Kaehler non Kaehler manifold is the six dimensional sphere $S^{6}$. It has an almost complex structure $J$ defined by vector cross product in the space of purely imaginary Cayley numbers which satisfy the condition (2.2).

There is a more general class of almost Hermitian manifolds than nearly Kaehler manifolds, known as RK-manifolds. These are defined as follows.

A RK-manifold $(\bar{M}, J, g, \bar{\nabla})$ is an almost Hermitian manifold for which the curvature tensor $\bar{R}$ is invariant under $J$, i.e.,

$$
\bar{R}(J U, J V, J W, J Z)=\bar{R}(U, V, W, Z)
$$

for any $U, V, W, Z \in T \bar{M}$.

An almost Hermitian manifold $\bar{M}$ is of pointwise constant type if for any $x \in \bar{M}$ and $U \in T_{x} \bar{M}$

$$
\lambda(U, V)=\lambda(U, W),
$$

where $\lambda(U, V)=\bar{R}(U, V, J U, J V)-\bar{R}(U, V, U, V)$ with $V$ and $W$ being tangent vectors at $x$, orthogonal to $U$ and $J U$. The manifold $\bar{M}$ is said to be of constant type if for any unit vectors $U, V \in T \bar{M}$ with $g(U, V)=g(J U, V)=0, \lambda(U, V)$ is a constant function. Then we have: 
Theorem 2.1 ([15]). Let $\bar{M}$ be an RK-manifold. Then $\bar{M}$ is of pointwise constant type if and only if there exists a function $\alpha$ on $\bar{M}$ such that

$$
\lambda(U, V)=\alpha\left[g(U, U) g(V, V)-(g(U, V))^{2}-(g(U, J V))^{2}\right]
$$

for any $U, V \in T \bar{M}$. Moreover, $\bar{M}$ is of constant type if and only if the above equality holds for a constant $\alpha$. In this case, $\alpha$ is the constant type of $\bar{M}$.

A generalized complex space form is an RK-manifold of constant holomorphic sectional curvature and of constant type. A generalized complex space form of constant holomorphic sectional curvature $c$ and of constant type $\alpha$ is denoted by $\bar{M}(c, \alpha)$. Each complex space form is a generalized complex space form. The converse is not true. The sphere $S^{6}$ endowed with the standard nearly Kaehler structure is an example of a generalized complex space form which is not a complex space form.

Let $\bar{M}(c, \alpha)$ be a generalized complex space form of constant holomorphic sectional curvature $c$ and of constant type $\alpha$. Then the curvature tensor $\bar{R}$ of $\bar{M}(c, \alpha)$ has the following expression

$$
\begin{aligned}
\bar{R}(U, V) W= & \frac{c+3 \alpha}{4}[g(V, W) U-g(U, W) V] \\
& +\frac{c-\alpha}{4}[g(U, J W) J V-g(V, J W) J U \\
& +2 g(U, J V) J W] .
\end{aligned}
$$

Let $M$ be a submanifold of an almost Hermitian manifold $\bar{M}$. Then we denote the induced metric on $M$ by the same symbol $g$ whereas the induced Riemannian connection on $M$ by $\nabla$. With these notations, Gauss and Weingarten formulae are written as

$$
\begin{aligned}
& \bar{\nabla}_{U} V=\nabla_{U} V+h(U, V), \\
& \bar{\nabla}_{U} N=-A_{N} U+\nabla_{U}^{\perp} N
\end{aligned}
$$

for each $U, V \in T M$ and $N \in T^{\perp} M$, where $\nabla^{\perp}$ denotes the induced connection on the normal bundle $T^{\perp} M . h$ and $A_{N}$ are the second fundamental form and the shape operator of the immersion of $M$ into $\bar{M}$. They are related by

$$
g(h(U, V), N)=g\left(A_{N} U, V\right) .
$$

For any $U \in T(M)$ and $N \in T^{\perp} M$, we write

$$
\begin{aligned}
& J U=P U+F U, \\
& J N=t N+f N,
\end{aligned}
$$

where $P U$ and $t N$ are the tangential components of $J U$ and $J N$, respectively whereas $F U$ and $f N$ are the normal components of $J U$ and $J N$, respectively.

The covariant differentiation of the tensors $P, F, t$, and $f$ are defined as

$$
\left(\bar{\nabla}_{U} P\right) V=\nabla_{U} P V-P \nabla_{U} V
$$




$$
\begin{gathered}
\left(\bar{\nabla}_{U} F\right) V=\nabla_{U}^{\perp} F V-F \nabla_{U} V, \\
\left(\bar{\nabla}_{U} t\right) N=\nabla_{U} t N-t \nabla_{U}^{\perp} N, \\
\left(\bar{\nabla}_{U} f\right) N=\nabla_{U}^{\perp} f N-f \nabla_{U}^{\perp} N .
\end{gathered}
$$

On the other hand the covariant derivative of the second fundamental form $h$ is defined as

$$
\left(\bar{\nabla}_{U} h\right)(V, W)=\nabla_{U}^{\frac{1}{U}} h(V, W)-h\left(\nabla_{U} V, W\right)-h\left(V, \nabla_{U} W\right)
$$

for any $U, V, W \in T M$. Let $\bar{R}$ and $R$ be the curvature tensors of the connections $\bar{\nabla}$ and $\nabla$ on $\bar{M}$ and $M$, respectively. Then the equations of Gauss and Codazzi are given by

$$
\begin{aligned}
\bar{R}(U, V, W, Z)= & R(U, V, W, Z)-g(h(U, W), h(V, Z)) \\
& +g(h(U, Z), h(V, W)), \\
{[\bar{R}(U, V) W]^{\perp}=} & \left(\bar{\nabla}_{U} h\right)(V, W)-\left(\bar{\nabla}_{V} h\right)(U, W) .
\end{aligned}
$$

A submanifold $M$ of $\bar{M}$ is said to be a $C R$-submanifold if there exists on $M$, a differentiable holomorphic distribution $D$ such that its orthogonal complementary distribution $D^{\perp}$ is totally real, i.e., $J D_{x} \subset T_{x}(M)$ and $J D_{x}^{\perp} \subset T_{x}^{\perp}(M)$ for each $x \in M$.

For a CR-submanifold of an almost Hermitian manifold $\bar{M}$, we have

$$
\begin{gathered}
T M=D \oplus D^{\perp}, \\
T^{\perp} M=J D^{\perp} \oplus \mu,
\end{gathered}
$$

where $\mu$ denotes the orthogonal complementary distribution of $J D^{\perp}$ and is an invariant normal subbundle of $T^{\perp} M$ under $J$.

The orthogonal projections on $T M$ are denoted by $B$ and $C$, i.e., for any $U \in T M$

$$
U=B U+C U,
$$

where $B U \in D$ and $C U \in D^{\perp}$. It is straight forward to observe that
(a) $P C=0$,
(b) $F B=0$,
(c) $t\left(T^{\perp} M\right)=D^{\perp}$,
(d) $\quad f\left(T^{\perp} M\right) \subset \mu$.

Furthermore, for $U, V \in T M$ if we denote by $\mathcal{P}_{U} V$ and $\mathcal{Q}_{U} V$, the tangential and normal parts of $\left(\bar{\nabla}_{U} J\right) V$, then by making use of (2.4)-(2.10), we obtain

$$
\begin{gathered}
\mathcal{P}_{U} V=\left(\bar{\nabla}_{U} P\right) V-A_{F V} U-t h(U, V), \\
\mathcal{Q}_{U} V=\left(\bar{\nabla}_{U} F\right) V+h(U, P V)-f h(U, V) .
\end{gathered}
$$

Similarly, for $N \in T^{\perp} M$, the tangential and normal parts of $\left(\bar{\nabla}_{U} J\right) N$ are respectively given by

$$
\mathcal{P}_{U} N=\left(\bar{\nabla}_{U} t\right) N+P A_{N} U-A_{f N} U,
$$




$$
\mathcal{Q}_{U} N=\left(\bar{\nabla}_{U} f\right) N+h(t N, U)+F A_{N} U .
$$

Let $\left(N_{1}, g_{1}\right)$ and $\left(N_{2}, g_{2}\right)$ be two Riemannian manifolds and $f$, a positive differentiable function on $N_{1}$. The warped product of $N_{1}$ and $N_{2}$ is the Riemannian manifold

$$
M=N_{1} \times_{f} N_{2}=\left(N_{1} \times N_{2}, g\right),
$$

where $g=g_{1}+f^{2} g_{2}$ [1]. $N_{1}$ is called the base of $M$, and $N_{2}$ the fibre.

For a warped product manifold $N_{1} \times_{f} N_{2}$, we denote by $D_{1}$ and $D_{2}$ the distributions defined by the vectors tangent to the leaves and fibers respectively. In other words, $D_{1}$ is obtained by the tangent vectors of $N_{1}$ via the horizontal lift and $D_{2}$ is obtained by the tangent vectors of $N_{2}$ via the vertical lift. In case of CR-warped product submanifolds $D_{1}$ and $D_{2}$ are replaced by $D$ and $D^{\perp}$ respectively.

A warped product $N_{1} \times_{f} N_{2}$ is said to be a trivial warped product if its warping function $f$ is constant. A trivial warped product $N_{1} \times_{f} N_{2}$ is nothing but a Riemannian product $N_{1} \times N_{2}^{f}$, where $N_{2}^{f}$ is the Riemannian manifold with Riemannian metric $f^{2} g_{N_{2}}$ which is homothetic to the original metric $g_{N_{2}}$ of $\mathrm{N}_{2}$.

\section{Some basic results}

A submanifold $M$ of an almost Hermitian manifold $\bar{M}$ is said to be a CRproduct submanifold if $M$ is locally a Riemannian product of a holomorphic submanifold $N_{T}$ and a totally real submanifold $N_{\perp}$. Thus a CR-submanifold of an almost Hermitian manifold is a CR-product if and only if both the distributions $D$ and $D^{\perp}$ on $M$ are integrable and their leaves are totally geodesic in $M$. It is proved that a CR-product in a complex space form is a product of a holomorphic submanifold and a totally real submanifold of complex linear subspaces and there do not exist CR-products in complex hyperbolic spaces. Moreover, CR-submanifolds in complex projective spaces $C P^{h+p+h p}$ are obtained from Segre imbedding in a natural way (cf. [6]).

For a CR-submanifold of a Kaehler manifold, Chen [2] proved:

Theorem 3.1 ([2]). A CR-submanifold of a Kaehler manifold is a CR-product if and only if $\bar{\nabla} P=0$ or equivalently $A_{J D^{\perp}} D=0$.

K. A. Khan et.al [10] worked out conditions for the two distributions on a CR-submanifold of a nearly Kaehler manifold to be integrable and parallel, which led to a characterization for a CR-submanifold to be a CR-product in a nearly Kaehler manifold. We recall:

Proposition 3.1 ([10]). The holomorphic distribution D on a CR-submanifold $M$ of a nearly Kaehler manifold is integrable if and only if

$$
\mathcal{Q}_{X} Y=0 \quad \text { and } \quad h(X, J Y)=h(J X, Y)
$$

for each $X, Y$ in $D$. 
Proposition 3.2 ([10]). The totally real distribution $D^{\perp}$ on a CR-submanifold $M$ of a nearly Kaehler manifold is integrable if and only if

$$
g\left(\mathcal{P}_{Z} W, X\right)=0, \quad \text { or equivalently, } \quad g\left(A_{J Z} W, X\right)=g\left(A_{J W} Z, X\right) .
$$

CR-submanifolds which are warped products have the forms $N_{\perp} \times_{f} N_{T}$ and $N_{T} \times{ }_{f} N_{\perp}$. These warped product submanifolds are known as warped product CR-submanifold and CR-warped product submanifolds respectively. Chen [4] proved that warped product CR-submanifolds of a Kaehler manifold are trivial, i.e., they are simply CR-products. Recently, the result is extended to the setting of nearly Kaehler manifolds, i.e., warped product CR-submanifolds of nearly Kaehler manifolds are CR-products (cf. [12]). However, many examples of CR-warped product submanifolds of a Kaehler manifold are provided in [4].

Earlier, K. Sekigawa [14] while studying submanifolds of $S^{6}$ proved:

Theorem 3.2 ([14]). There does not exist a CR-product in $S^{6}$.

This paved way to study CR-warped product submanifolds in $S^{6}$ and in a nearly Kaehler manifold in general. Sekigawa [14] obtained an example of a CR-warped product submanifold in $S^{6}$. For a more general case, N. Ejiri [7] provided a categorical answer to the existence of warped product submanifolds in $S^{6}$. He proved:

Theorem $3.3([7])$. There exist countably many immersions of $S^{1} \times S^{n-1}$ into $S^{n+1}$ such that the induced metric on it is a warped product metric of constant scaler curvature $n(n-1)$.

Moreover, every Riemannian manifold of constant scalar curvature $c$ can be locally expressed as a warped product whose warping function satisfies $\triangle f=$ $c f$. For example, $S^{n}(1)$ is locally isometric to $(-\pi / 2, \pi / 2) \times \cos t S^{n-1}(1), R^{n}$ is isometric to $(0, \infty) \times{ }_{x} S^{n-1}(1)$ and $H^{n-1}(-1)$ is locally isometric to $R \times{ }_{e^{x}} R^{n-1}$.

As far as the intrinsic geometry of a warped product manifold is concerned Bishop and O'Neill obtained:

Theorem 3.4 ([1]). Let $M=N_{1} \times_{f} N_{2}$ be a warped product manifold. Then

(i) $N_{1}$ is a totally geodesic submanifold of $M$ and

(ii) $\mathrm{N}_{2}$ is a totally umbilical submanifold of $M$.

Moreover,

$$
\nabla_{U} V=\nabla_{V} U=(U \ln f) V
$$

and

$$
\operatorname{nor}\left(\nabla_{V} W\right)=\frac{-g(V, W)}{f} \nabla f
$$

for any $U \in D_{1}$ and $V, W \in D_{2}$, where nor $\left(\nabla_{V} W\right)$ denotes the component of $\nabla_{V} W$ in $D_{1}$ and $\nabla f$ denotes the gradient of $f$ and is defined as

$$
g(\nabla f, U)=U f
$$




\section{CR-warped products and the canonical structures}

In [2], B. Y. Chen obtained various conditions under which a CR-submanifold reduces to a CR-product. In particular, he showed that a CR-submanifold of a Kaehler manifold is a CR-product if and only if $\bar{\nabla} P=0$. Later he extended the characterization while studying the impact of parallelism of the $(1,1)$ tensor field $P$ on an arbitrary submanifold of a Kaehler manifold. In this case, he proved that $\nabla P=0$ if and only if the submanifold is locally a Riemannian product of submanifolds which are either holomorphic or totally real or Kaehlerian slant (cf. [3]). As warped product manifolds are generalized version of product manifolds, it is natural to seek analogous conditions under which a CR-submanifold is a CR-warped product submanifold. In this section, we have obtained necessary and sufficient conditions involving $P$ and $F$, forcing a CR-submanifold to be locally a CR-warped product submanifold. To prove the main theorems, we first obtain some useful relations.

Lemma 4.1. Let $M$ be a CR-warped product submanifold $N_{T} \times_{f} N_{\perp}$ of an almost Hermitian manifold $\bar{M}$. Then

$$
\begin{gathered}
\left(\bar{\nabla}_{Z} P\right) X=(P X \ln f) Z, \\
\left(\bar{\nabla}_{U} P\right) Z=g(C U, Z) P(\nabla \ln f)
\end{gathered}
$$

for each $U \in T M, X \in D$ and $Z \in D^{\perp}$, where $\nabla \ln f$ denotes the gradient of $\ln f$.

Proof. By formula (3.1)

$$
\nabla_{X} Z=\nabla_{Z} X=(X \ln f) Z
$$

and therefore by making use of formulae (2.9) and (2.19), we obtain (4.1). On the other hand as $P U \in D$ for any $U \in T M$, by formulae (2.9) and (2.19), we find that $\left(\bar{\nabla}_{U} P\right) Z \in D$. Now for any $X \in D$,

$$
\begin{aligned}
g\left(\left(\bar{\nabla}_{U} P\right) Z, X\right) & =g\left(\nabla_{U} Z, P X\right) \\
& =-g\left(Z, \nabla_{U} P X\right) \\
& =-g\left(Z, \nabla_{C U} P X\right) \\
& =-(P X \ln f) g(C U, Z),
\end{aligned}
$$

which on taking account of (3.3) gives

$$
\left(\bar{\nabla}_{U} P\right) Z=g(C U, Z) P(\nabla \ln f) .
$$

This proves the lemma.

Theorem 4.1. Let $\bar{M}$ be a nearly Kaehler manifold and $M$ be a CR-submanifold of $\bar{M}$ with integrable distributions $D$ and $D^{\perp}$. Then $M$ is a CR-warped product submanifold of $\bar{M}$ if and only if

$$
\left(\bar{\nabla}_{U} P\right) U=(P U \mu) C U+\|C U\|^{2} J \nabla \mu
$$


for each $U \in T M$ and $\mu$ a $C^{\infty}$-function on $M$ satisfying $Z \mu=0$ for each $Z \in D^{\perp}$.

Proof. The relation (4.3) is equivalent to

$$
\left(\bar{\nabla}_{U} P\right) V+\left(\bar{\nabla}_{V} P\right) U=(P U \mu) C V+(P V \mu) C U+2 g(C U, C V) J \nabla \mu,
$$

with $U, V \in T M$. Let $M$ be a CR-warped product submanifold of $\bar{M}$. For any $U \in T M$, we may write

$$
\left(\bar{\nabla}_{U} P\right) U=\left(\bar{\nabla}_{B U} P\right) B U+\left(\bar{\nabla}_{C U} P\right) B U+\left(\bar{\nabla}_{U} P\right) C U .
$$

The first term in the right hand side of (4.5) is zero as $N_{T}$ is totally geodesic in $M$ and by Lemma 4.1 ,

$$
\begin{aligned}
& \left(\bar{\nabla}_{C U} P\right) B U=(P U \ln f) C U, \\
& \left(\bar{\nabla}_{U} P\right) C U=\|C U\|^{2} P \nabla \ln f .
\end{aligned}
$$

From (4.5), (4.6) and (4.7),

$$
\left(\bar{\nabla}_{U} P\right) U=(P U \ln f) C U+\|C U\|^{2} P \nabla \ln f .
$$

Conversely, suppose that $M$ is a CR-submanifold of $\bar{M}$ with $D$ and $D^{\perp}$ involutive on $M$ and such that (4.3) holds for a $C^{\infty}$-function $\mu$ on $M$ with $Z \mu=0$ for each $Z \in D^{\perp}$. It follows from (4.4) that

$$
\left(\bar{\nabla}_{X} P\right) Y+\left(\bar{\nabla}_{Y} P\right) X=0
$$

for each $X, Y \in D$. Further, as $\bar{M}$ is nearly Kaehler, it follows from (2.2) that

$$
\mathcal{P}_{U} V+\mathcal{P}_{V} U=0, \quad \text { and } \mathcal{Q}_{U} V+\mathcal{Q}_{V} U=0
$$

for each $U, V \in T M$. From (4.8) and the first part of (4.9), it follows on using (2.20) that $\operatorname{th}(X, Y)=0$. That means

$$
h(X, Y) \in \mu
$$

for each $X, Y \in D$. Now, for $Z \in D^{\perp}$

$$
g\left(\nabla_{X} Y, Z\right)=g\left(J \bar{\nabla}_{X} Y, J Z\right) .
$$

As $D$ is involutive, on using Proposition 3.1, formula (2.2) and the observation (4.10), the above equation yields

$$
g\left(\nabla_{X} Y, Z\right)=0 .
$$

This proves that the leaves of $D$ are totally geodesic in $M$. On the other hand by formulae (2.21) and (4.9)

$$
\left(\bar{\nabla}_{X} P\right) Z+\left(\bar{\nabla}_{Z} P\right) X=A_{F Z} X+2 t h(X, Z),
$$

whereas by (4.4),

$$
\left(\bar{\nabla}_{X} P\right) Z+\left(\bar{\nabla}_{Z} P\right) X=(P X \mu) Z
$$

for each $X \in D$ and $Z \in D^{\perp}$. From (4.11) and (4.12),

$$
g\left(A_{F Z} X, W\right)-2 g(h(X, Z), J W)=(P X \mu) g(Z, W)
$$


for each $W \in D^{\perp}$. As $D^{\perp}$ is involutive, in view of Proposition 3.2, (4.13) gives

$$
g(h(X, Z), J W)=-(P X \mu) g(Z, W)
$$

or

$$
g\left(\nabla_{Z} J X-\mathcal{P}_{Z} X, W\right)=(P X \mu) g(Z, W) .
$$

On taking account of Proposition 3.2, the above relation yields

$$
g\left(\nabla_{Z} W, J X\right)=-(P X \mu) g(Z, W) .
$$

Let $N_{T}$ and $N_{\perp}$ denote the leaves of $D$ and $D^{\perp}$ respectively. If $h^{\prime}$ denotes the second fundamental form of the immersion of $N_{\perp}$ into $M$, then (4.14) can be written as

$$
g\left(h^{\prime}(Z, W), J X\right)=(J X \mu) g(Z, W)=g(\nabla \mu, J X) g(Z, W) .
$$

Thus we obtain

$$
h^{\prime}(Z, W)=g(Z, W) \nabla \mu .
$$

Thus each leaf $N_{\perp}$ of $D^{\perp}$ is totally umbilical in $M$ and as $Z \mu=0$ for each $Z \in D^{\perp}$, the mean curvature vector $\nabla \mu$ is parallel on $N_{\perp}$, i.e., $N_{\perp}$ is an extrinsic sphere in $M$. Hence by virtue of a theorem in [9], which states that "If the tangent bundle of a Riemannian manifold $M$ splits into an orthogonal sum $T M=E_{0} \oplus E_{1}$ of non trivial vector subbundles such that $E_{1}$ is spherical and its orthogonal complement $E_{0}$ is autoparallel, then the manifold $M$ is locally isometric to a warped product $M_{0} \times_{f} M_{1}$ ", we get that $M$ is locally a CR-warped product submanifold $N_{T} \times_{f} N_{\perp}$, where $f=e^{\mu}$.

In terms of the structure $F$, we have:

Theorem 4.2. Let $M$ be a CR-submanifold of a nearly Kaehler manifold $\bar{M}$ with totally real distribution being involutive. Then $M$ is locally a CR-warped product submanifold if and only if

$$
g\left(\left(\bar{\nabla}_{U} F\right) V, J W\right)=g\left(\mathcal{Q}_{C U} C V, J W\right)-(B V \mu) g(C U, W)
$$

for each $U, V \in T M$ and $W \in D^{\perp}$, where $\mu$ is a $C^{\infty}$-function on $M$ such that $Z \mu=0$ for each $Z \in D^{\perp}$.

Proof. Let $M$ be a CR-warped product submanifold $N_{T} \times_{f} N_{\perp}$. Then for any $X, Y \in D$ and $W \in D^{\perp}$, by formula (2.10)

$$
g\left(\left(\bar{\nabla}_{X} F\right) Y, J W\right)=-g\left(F \nabla_{X} Y, J W\right)=-g\left(\nabla_{X} Y, W\right) .
$$

Therefore, as $N_{T}$ is totally geodesic in $M$

$$
g\left(\left(\bar{\nabla}_{X} F\right) Y, J W\right)=0 .
$$

On the other hand, for any $X \in D$ and $Z, W \in D^{\perp}$, by formula (2.21)

$$
\begin{aligned}
\left.g\left(\bar{\nabla}_{X} F\right) Z, J W\right) & =g\left(\mathcal{Q}_{X} Z+f h(X, Z), J W\right) \\
& =g\left(\mathcal{Q}_{X} Z, J W\right) \\
& =-g\left(\mathcal{P}_{Z} J X, W\right) \\
& =g\left(J X, \mathcal{P}_{Z} W\right) .
\end{aligned}
$$


Thus, by Proposition 3.2, we have

$$
\left.g\left(\bar{\nabla}_{X} F\right) Z, J W\right)=0 .
$$

Further,

$$
g\left(\left(\bar{\nabla}_{Z} F\right) X, J W\right)=-g\left(F \nabla_{Z} X, J W\right),
$$

which on using (3.1) gives that

$$
g\left(\left(\bar{\nabla}_{Z} F\right) X, J W=-(X \ln f) g(Z, W),\right.
$$

and for any $W^{\prime} \in D^{\perp}$, by formula (2.21) we have

$$
\begin{aligned}
g\left(\left(\bar{\nabla}_{Z} F\right) W^{\prime}, J W\right) & =g\left(\mathcal{Q}_{Z} W^{\prime}+f h\left(Z, W^{\prime}\right), J W\right) \\
& =g\left(\mathcal{Q}_{Z} W^{\prime}, J W\right) .
\end{aligned}
$$

Now, for $U, V \in T M$ we may write

$$
\begin{aligned}
g\left(\left(\bar{\nabla}_{U} F\right) V, J W\right)= & g\left(\left(\bar{\nabla}_{B U} F\right) B V, J W\right)+g\left(\left(\bar{\nabla}_{B U} F\right) C V, J W\right) \\
& +g\left(\left(\bar{\nabla}_{C U} F\right) B V, J W\right)+g\left(\left(\bar{\nabla}_{C U} F\right) C V, J W\right) .
\end{aligned}
$$

The first two terms in the right hand side of the above equation are zero in view of (4.16) and (4.17) and the remaining terms in view of (4.18) and (4.19) yield (4.15).

Conversely, suppose that $M$ is a CR-submanifold of a nearly Kaehler manifold $\bar{M}$ such that (4.15) holds. Then obviously

$$
g\left(\left(\bar{\nabla}_{X} F\right) Y, J W\right)=0
$$

for each $X, Y \in D$ and $W \in D^{\perp}$. Therefore $g\left(\nabla_{X} Y, W\right)=0$, that means $D$ is integrable and its leaves are totally geodesic in $M$. Now, for any $Z, W \in D^{\perp}$, by (4.15) we have

$$
g\left(\left(\bar{\nabla}_{Z} F\right) X, J W\right)=-(X \mu) g(Z, W)
$$

or

$$
g\left(\nabla_{Z} W, X\right)=-(X \mu) g(Z, W) .
$$

Let $N_{\perp}$ be a leaf of $D^{\perp}$. If $\nabla^{\prime}$ denotes the induced Riemannian connection on $N_{\perp}$ and $h^{\prime}$, the second fundamental form of the immersion of $N_{\perp}$ into $M$, then the last equation in view of Gauss formula is written as

$$
g\left(X, \nabla_{Z}^{\prime} W+h^{\prime}(Z, W)\right)=-(X \mu) g(Z, W)
$$

or

$$
\begin{aligned}
g\left(X, h^{\prime}(Z, W)\right) & =-(X \mu) g(Z, W) \\
& =-g(X, \nabla \mu) g(Z, W),
\end{aligned}
$$

or

$$
h^{\prime}(Z, W)=-g(Z, W) \nabla \mu .
$$

This shows that $N_{\perp}$ is totally umbilical in $M$ and in view of the condition that $Z \mu=0$ for each $Z \in D^{\perp}, \nabla \mu$ is defined on $N_{T}$ only, i.e., the mean curvature $\nabla \mu$ is parallel on $N_{\perp}$. In other words, $N_{\perp}$ is an extrinsic sphere. 
Hence, by a similar argument as given in Theorem 4.1, $M$ is locally isometric to a warped product submanifold with a warping function $f=e^{\mu}$.

Let $M=N_{T} \times_{f} N_{\perp}$ be a CR-warped product submanifold of a nearly Kaehler manifold $\bar{M}$. In view of the decomposition (2.17), we may write

$$
h(U, V)=h_{J D^{\perp}}(U, V)+h_{\mu}(U, V)
$$

for each $U, V \in T M$, where $h_{J D^{\perp}}(U, V) \in J D^{\perp}$ and $h_{\mu}(U, V) \in \mu$.

If $\left\{e_{1}, e_{2}, \ldots, e_{n}\right\}$ be a local orthonormal frame of vector fields on $M$, then we define

$$
\|h\|^{2}=\sum_{i, j=1}^{n} g\left(h\left(e_{i}, e_{j}\right), h\left(e_{i}, e_{j}\right)\right),
$$

and for a differentiable function $f$ on $M$, the Laplacian $\triangle f$ of $f$ is defined by

$$
\triangle f=\sum_{j=1}^{n}\left[\left(e_{j}\left(e_{j} f\right)-\nabla_{e_{j}} e_{j}\right) f\right] .
$$

Proposition 4.1. Let $M$ be a CR-warped product submanifold of a nearly Kaehler manifold $\bar{M}$. Then

(i) $h_{J D^{\perp}}(J X, Z)=(X \ln f) J Z$,

(ii) $g\left(\mathcal{Q}_{Z} X, J W\right)=0$,

(iii) $g(h(J X, Z), J h(X, Z))=\left\|h_{\mu}(X, Z)\right\|^{2}-g\left(\mathcal{Q}_{X} Z, J h_{\mu}(X, Z)\right)$

for each $X \in D$ and $Z, W \in D^{\perp}$.

Proof. By Gauss formula,

$$
\begin{aligned}
h(J X, Z) & =\bar{\nabla}_{Z} J X-\nabla_{Z} J X \\
& =\left(\bar{\nabla}_{Z} J\right) X+J \nabla_{Z} X+J h(X, Z)-\nabla_{Z} J X .
\end{aligned}
$$

Thus, on using (3.1), we obtain

$$
h(J X, Z)=\mathcal{P}_{Z} X+\mathcal{Q}_{Z} X+(X \ln f) J Z+J h(X, Z)-(J X \ln f) Z .
$$

Comparing tangential parts in (4.24), we get

$$
\mathcal{P}_{Z} X=(J X \ln f) Z-J h_{J D^{\perp}}(X, Z) .
$$

Taking product with $W$ in both sides, yields

$$
g\left(\mathcal{P}_{Z} X, W\right)=(J X \ln f) g(Z, W)+g\left(h_{J D^{\perp}}(X, Z), J W\right) .
$$

The left hand side of the last equation is zero in view of Proposition 3.2 and thus the equation reduces to

$$
g(h(X, Z), J W)=-(J X \ln f) g(Z, W),
$$

or equivalently,

$$
h_{J D^{\perp}}(J X, Z)=(X \ln f) J Z .
$$

This proves (i). Now, on comparing the normal parts in (4.24), we get

$$
h(J X, Z)=\mathcal{Q}_{Z} X+(X \ln f) J Z+J h_{\mu}(X, Z)
$$


or

$$
h(J X, Z)-J h_{\mu}(X, Z)=\mathcal{Q}_{Z} X+(X \ln f) J Z .
$$

Taking product with $J W$ in (4.26) and using (4.25), we obtain statement (ii) of the proposition, i.e.,

$$
g\left(\mathcal{Q}_{Z} X, J W\right)=0 .
$$

Now, by (4.26)

$$
g(h(J X, Z), J h(X, Z))=g\left(J h_{\mu}(X, Z)+\mathcal{Q}_{Z} X, J h_{\mu}(X, Z)\right) .
$$

Or,

$$
g(h(J X, Z), J h(X, Z))=\left\|h_{\mu}(X, Z)\right\|^{2}-g\left(\mathcal{Q}_{X} Z, J h_{\mu}(X, Z)\right) .
$$

This proves the proposition completely.

For CR-warped products in nearly Kaehler manifolds, we have the following:

Theorem 4.3. Let $M=N_{T} \times{ }_{f} N_{\perp}$ be a CR-warped product submanifold of a nearly Kaehler manifold $\bar{M}$. Then we have

(i) The squared norm of the second fundamental form satisfies

$$
\|h\|^{2} \geq 2 q\|\nabla \ln f\|^{2}
$$

where $\nabla \ln f$ is the gradient of $\ln f$ and $q$ is the dimension of $N_{\perp}$.

(ii) If the equality sign in (4.28) holds identically, then $N_{T}$ is totally geodesic submanifold of $\bar{M}, N_{\perp}$ a totally umbilical submanifold of $\bar{M}$ and $M$ is a minimal submanifold of $\bar{M}$.

Proof. Let $\left\{X_{1}, X_{2}, \ldots, X_{p}, X_{p+1}=J X_{1}, \ldots, X_{2 p}=J X_{p}\right\}$ be a local orthonormal frame of vector fields on $N_{T}$ and $\left\{Z_{1}, Z_{2}, \ldots, Z_{q}\right\}$ a local orthonormal frame on $N_{\perp}$. Then by definition

$$
\begin{aligned}
\|h\|^{2}= & \sum_{i, j=1}^{2 p} g\left(h\left(X_{i}, X_{j}\right), h\left(X_{i}, X_{j}\right)\right)+\sum_{i=1}^{2 p} \sum_{r=1}^{q} g\left(h\left(X_{i}, Z_{r}\right), h\left(X_{i}, Z_{r}\right)\right) \\
& +\sum_{r, s=1}^{q} g\left(h\left(Z_{r}, Z_{s}\right), h\left(Z_{r}, Z_{s}\right)\right) .
\end{aligned}
$$

Thus,

$$
\|h\|^{2} \geq \sum_{i=1}^{2 p} \sum_{r=1}^{q} g\left(h\left(X_{i}, Z_{r}\right), h\left(X_{i}, Z_{r}\right)\right)
$$

or

$$
\begin{aligned}
\|h\|^{2} & \geq \sum_{i=1}^{2 p} \sum_{r=1}^{q}\left(X_{i} \ln f\right)^{2} g\left(Z_{r}, Z_{r}\right) \\
& \geq 2 q\|\nabla \ln f\|^{2} .
\end{aligned}
$$


This verifies the assertion (i). If the equality sign in (4.28) holds, then by (4.29) and (4.25), we obtain

$$
h(D, D)=0, \quad h\left(D^{\perp}, D^{\perp}\right)=0 \quad \text { and } \quad h\left(D, D^{\perp}\right) \subset J D^{\perp},
$$

since $N_{T}$ is a totally geodesic submanifold of $M$, the first condition in (4.30) implies that $N_{T}$ is totally geodesic in $\bar{M}$. Moreover as $N_{\perp}$ is totally umbilical in $M$, the second condition in (4.30) implies that $N_{\perp}$ is totally umbilical in $\bar{M}$. It also follows from (4.30) that $M$ is minimal in $\bar{M}$.

\section{CR-warped products in a generalized complex space form}

Throughout the section, we denote by $\bar{M}(c, \alpha)$ a generalized complex space form of constant curvature $c$ and of constant type $\alpha$. Let $M=N_{T} \times_{f} N_{\perp}$ be a CR-warped product submanifold of $\bar{M}(c, \alpha)$.

Various inequalities involving the squared norm of the second fundamental form as well as that of the mean curvature vector of a CR-warped product submanifolds in real and complex space forms are obtained (cf. [5], [6] etc). For example, every CR-warped product $N_{T} \times{ }_{f} N_{\perp}$ in a complex space form $\bar{M}(4 c)$ satisfies the general inequality

$$
\|h\|^{2} \geq 2 q\left\{\|\nabla \ln f\|^{2}+\triangle \ln f\right\}+4 p q c,
$$

where $p=\operatorname{dim}_{C} N_{T}, q=\operatorname{dim}_{R} N_{\perp}[6]$.

Now, as the class of generalized complex space forms includes nearly Kaehler manifolds of constant holomorphic sectional curvature, it is interesting to study similar estimates in the setting of generalized complex space forms. To this end we prove:

Theorem 5.1. Let $M=N_{T} \times{ }_{f} N_{\perp}$ be a CR-warped product submanifold of a generalized complex space form $\bar{M}(c, \alpha)$. Then we have

$$
\|h\|^{2} \geq 2 q\left\{\|\nabla \ln f\|^{2}+\frac{1}{2}(\triangle \ln f)+\frac{p(c-\alpha)}{4}\right\},
$$

where $h$ denotes the second fundamental form of the immersion of $M$ into $\bar{M}(c, \alpha), \nabla \ln f$ is the gradient of $\ln f, \triangle \ln f$ is the Laplacian of $\ln f, 2 p$ and $q$ are the real dimensions of $N_{T}$ and $N_{\perp}$ respectively.

Proof. For $X \in D$ and $Z \in D^{\perp}$, by formula (2.3), we have

$$
\bar{R}(X, J X, Z, J Z)=\left(\frac{\alpha-c}{2}\right) g(X, X) g(Z, Z) .
$$

On the other hand by Codazzi equation

$$
\begin{aligned}
\bar{R}(X, J X, Z, J Z)= & g\left(\nabla_{X}^{\perp} h(J X, Z), J Z\right)-g\left(h\left(\nabla_{X} J X, Z\right), J Z\right) \\
& -g\left(h\left(J X, \nabla_{X} Z\right), J Z\right)-g\left(\nabla_{J X}^{\perp} h(X, Z), J Z\right) \\
& +g\left(h\left(\nabla_{J_{X}} X, Z\right), J Z\right)+g\left(h\left(X, \nabla_{J_{X}} Z\right), J Z\right) .
\end{aligned}
$$


Now,

$$
g\left(\nabla \frac{1}{X} h(J X, Z), J Z\right)=X g(h(J X, Z), J Z)-g\left(h(J X, Z), \bar{\nabla}_{X} J Z\right) .
$$

The first term in the right hand side of (5.5), on using formulae (4.25) and (3.1) takes the form

$$
X g(h(J X, Z), J Z)=\left(X(X \ln f)+2(X \ln f)^{2}\right) g(Z, Z),
$$

whereas the second term is written as

$$
\begin{aligned}
g\left(h(J X, Z), \bar{\nabla}_{X} J Z\right)= & g\left(h(J X, Z), \mathcal{Q}_{X} Z+J \nabla_{X} Z+J h(X, Z)\right) \\
= & g\left(h(J X, Z), \mathcal{Q}_{X} Z\right)+(X \ln f)^{2} g(Z, Z) \\
& +g(h(J X, Z), J h(X, Z)) .
\end{aligned}
$$

Making use of (4.27), the above equality takes the form

$$
\begin{aligned}
g\left(h(J X, Z), \bar{\nabla}_{X} J Z\right)= & g\left(h(J X, Z)-J h(X, Z), \mathcal{Q}_{X} Z\right) \\
& +(X \ln f)^{2} g(Z, Z)+\left\|h_{\mu}(X, Z)\right\|^{2} .
\end{aligned}
$$

On using (4.26), the above relation can be written as

$$
g\left(h(J X, Z), \bar{\nabla}_{X} J Z\right)=(X \ln f)^{2} g(Z, Z)+\left\|h_{\mu}(X, Z)\right\|^{2}-\left\|\mathcal{Q}_{X} Z\right\|^{2} .
$$

On substituting from (5.6) and (5.7), equation (5.5) yields

$$
\begin{aligned}
g\left(\nabla \frac{\perp}{X} h(J X, Z), J Z\right)= & \left(X(X \ln f)+(X \ln f)^{2}\right) g(Z, Z) \\
& +\left\|\mathcal{Q}_{X} Z\right\|^{2}-\left\|h_{\mu}(X, Z)\right\|^{2} .
\end{aligned}
$$

Similarly, we obtain

$$
\begin{aligned}
g\left(\nabla_{J X}^{\perp} h(X, Z), J Z\right)= & -\left(J X(J X \ln f)+(J X \ln f)^{2}\right) g(Z, Z) \\
& -\left\|\mathcal{Q}_{J X} Z\right\|^{2}+\left\|h_{\mu}(J X, Z)\right\|^{2} .
\end{aligned}
$$

By formulae (3.1) and (4.25), we have

$$
g\left(h\left(J X, \nabla_{X} Z\right), J Z\right)=(X \ln f)^{2} g(Z, Z),
$$

and

$$
g\left(h\left(X, \nabla_{J X} Z\right), J Z\right)=-(J X \ln f)^{2} g(Z, Z) .
$$

Further by making use of formula (3.1) and the fact that $N_{T}$ is totally geodesic in $M$, we get

$$
g\left(h\left(\nabla_{J X} X, Z\right), J Z\right)=-\left(J \nabla_{J X} X\right)(\ln f) g(Z, Z),
$$

and,

$$
g\left(h\left(\nabla_{X} J X, Z\right), J Z\right)=-\left(\left(J \nabla_{X} J X\right) \ln f\right) g(Z, Z)
$$


The right hand side of the above equation, on making use of the fact that $N_{T}$ is totally geodesic in $M$ and the formula (3.1) reduces to $-g\left(\nabla_{Z} J \nabla_{X} J X, Z\right)$. Thus, by using Gauss formula, we get

$$
\begin{aligned}
g\left(h\left(\nabla_{X} J X, Z\right), J Z\right)= & -g\left(\nabla_{X} X, \nabla_{Z} Z\right)-g\left(J h(X, J X), \nabla_{Z} Z\right) \\
= & \left(\left(\nabla_{X} X\right) \ln f\right) g(Z, Z)+\left(\left(\nabla_{J X} J X\right) \ln f\right) g(Z, Z) \\
& -\left(\left(J \nabla_{J X} X \ln f\right) g(Z, Z) .\right.
\end{aligned}
$$

Let $\left\{X_{1}, X_{2}, \ldots, X_{p}, X_{p+1}=J X_{1}, \ldots, X_{2 p}=J X_{p}\right\}$ and $\left\{Z_{1}, Z_{2}, \ldots, Z_{q}\right\}$ be a local frame of orthonormal vector fields on $N_{T}$ and $N_{\perp}$ respectively. Choosing $X, Z$ as basic vector fields and substituting from (5.8)-(5.13) into (5.4), we obtain

$$
\begin{aligned}
& \bar{R}\left(X_{i}, J X_{i}, Z_{r}, J Z_{r}\right) \\
= & \left(X_{i}\left(X_{i} \ln f\right)+J X_{i}\left(J X_{i} \ln f\right)\right) g\left(Z_{r}, Z_{r}\right) \\
& -\left(\left(\nabla_{X_{i}} X_{i}\right) \ln f+\left(\nabla_{J X_{i}} J X_{i}\right) \ln f\right) g\left(Z_{r}, Z_{r}\right) \\
& +\left\|\mathcal{Q}_{X_{i}} Z_{r}\right\|^{2}+\left\|\mathcal{Q}_{J X_{i}} Z_{r}\right\|^{2}-\left\|h_{\mu}\left(X_{i}, Z_{r}\right)\right\|^{2}-\left\|h_{\mu}\left(J X_{i}, Z_{r}\right)\right\|^{2} .
\end{aligned}
$$

Summing both sides over $i=1,2, \ldots, p$ and $r=1,2, \ldots, q$ and making use of (5.3) and (4.23), we obtain

$$
\frac{p q(\alpha-c)}{2}=\left\|\mathcal{Q}_{D} D^{\perp}\right\|^{2}-\left\|h_{\mu}\left(D, D^{\perp}\right)\right\|^{2}+q \triangle \ln f,
$$

where we have used the following notations

$$
\left\|\mathcal{Q}_{D} D^{\perp}\right\|^{2}=\sum_{i=1}^{2 p} \sum_{r=1}^{q}\left\|\mathcal{Q}_{X_{i}} Z_{r}\right\|^{2},
$$

and,

$$
\left\|h_{\mu}\left(D, D^{\perp}\right)\right\|^{2}=\sum_{i=1}^{2 p} \sum_{r=1}^{q}\left\|h_{\mu}\left(X_{i}, Z_{r}\right)\right\|^{2} .
$$

Further, if we denote $\sum_{i=1}^{2 p} \sum_{r=1}^{q}\left\|h_{J D^{\perp}}\left(X_{i}, Z_{r}\right)\right\|^{2}$ by $\left\|h_{J D^{\perp}}\left(D, D^{\perp}\right)\right\|^{2}$, then from (4.29), we have

$$
\left\|h_{J D^{\perp}}\left(D, D^{\perp}\right)\right\|^{2}=2 q\|\nabla \ln f\|^{2},
$$

whereas from (5.14), we have

$$
\left\|h_{\mu}\left(D, D^{\perp}\right)\right\|^{2}=\left\|\mathcal{Q}_{D} D^{\perp}\right\|^{2}+\frac{p q(c-\alpha)}{2}+q \triangle \ln f .
$$

On adding (5.15) and (5.16), we get

$$
\left\|h\left(D, D^{\perp}\right)\right\|^{2}=2 q\|\nabla \ln f\|^{2}+q \triangle \ln f+\left\|\mathcal{Q}_{D} D^{\perp}\right\|^{2}+\frac{p q(c-\alpha)}{2} .
$$


Hence, $\|h\|^{2}$ for the CR-warped product submanifold of a generalized complex space form $\bar{M}(c, \alpha)$ satisfy

$$
\|h\|^{2} \geq 2 q\left\{\|\nabla \ln f\|^{2}+\frac{1}{2} \triangle \ln f+\frac{p(c-\alpha)}{4}\right\} .
$$

The above inequality generalizes the inequality (5.1).

In particular for the CR-warped product of $S^{6}$, the above inequality reduces to

$$
\|h\|^{2} \geq 2 q\left\{\|\nabla \ln f\|^{2}+\frac{\triangle \ln f}{2}\right\},
$$

which improves the inequality (4.28).

\section{References}

[1] R. L. Bishop and B. O'Neill, Manifolds of negative curvature, Trans. Amer. Math. Soc. 145 (1969), 1-49.

[2] B. Y. Chen, CR-submanifolds of a Kaehler manifold. I, J. Differential Geom. 16 (1981), no. 2, 305-322.

[3] — Geometry of Slant Submanifolds, Katholieke Universiteit Leuven, Louvain, 1990.

[4] Geometry of warped product CR-submanifolds in Kaehler manifolds, Monatsh. Math. 133 (2001), no. 3, 177-195.

[5] __ On isometric minimal immersions from warped products into real space forms, Proc. Edinb. Math. Soc. (2) 45 (2002), no. 3, 579-587.

[6] _ Another general inequality for CR-warped products in complex space forms, Hokkaido Math. J. 32 (2003), no. 2, 415-444.

[7] N. Ejiri, Some compact hypersurfaces of constant scalar curvature in a sphere, J. Geom. 19 (1982), no. 2, 197-199.

[8] I. Hasegawa and I. Mihai, Contact CR-warped product submanifolds in Sasakian manifolds, Geom. Dedicata 102 (2003), 143-150.

[9] S. Hiepko, Eine innere Kennzeichnung der verzerrten Produkte, Math. Ann. 241 (1979), no. 3, 209-215.

[10] K. A. Khan, V. A. Khan, and S. I. Husain, On the integrability of the distributions on a CR-submanifold, An. Ştiinţ. Univ. Al. I. Cuza Iaşi Secţ. I a Mat. 38 (1992), no. 4, $367-378$.

[11] _ Totally umbilical CR-submanifolds of nearly Kähler manifolds, Geom. Dedicata 50 (1994), no. $1,47-51$.

[12] V. A. Khan, K. A. Khan, and Saraj Uddin, Warped product CR-submanifolds of a nearly Kaehler manifold, SUT Journal of Math. 43 (2007), no. 2, 201-213.

[13] B. Sahin, Nonexistence of warped product semi-slant submanifolds of Kaehler manifolds, Geom. Dedicata 117 (2006), 195-202.

[14] K. Sekigawa, Some CR-submanifolds in a 6-dimensional sphere, Tensor (N.S.) 41 (1984), no. 1, 13-20.

[15] F. Urbano, CR-submanifolds of nearly Kaehler manifolds, Doctorial Thesis, Granada, 1980. 
CR-WARPED PRODUCT SUBMANIFOLDS OF NEARLY KAEHLER MANIFOLDS 995

\section{Nadia S. Al-Luhaibi}

Department of Mathematics

King AbdulAziz University

P. O. Box 80015, Jeddah 21589, Saudi Arabia

E-mail address: rwhehotmail.com

Falleh R. Al-Solamy

Department of Mathematics

King AbdulAziz University

P. O. Box 80015, Jeddah 21589, Saudi Arabia

E-mail address: falleh@hotmail.com

VIQAR AZAM KHAN

Department of Mathematics

King AbdulAziz University

P. O. Box 80015, Jeddah 21589, Saudi Arabia

E-mail address: viqarster@gmail.com 